\title{
Quantum Correlations: Entropy, Wave/Corpuscle Dualism, Bell Inequality
}

\author{
Sebastiano Tosto* \\ ENEA Casaccia, via Anguillarese, Roma, Italy \\ Email: stosto@inwind.it, stosto44@gmail.com
}

How to cite this paper: Tosto, S. (2019) Quantum Correlations: Entropy, Wave/ Corpuscle Dualism, Bell Inequality. Open Journal of Physical Chemistry, 9, 60-87. https://doi.org/10.4236/ojpc.2019.92005

Received: April 15, 2019

Accepted: May 28, 2019

Published: May 31, 2019

Copyright $\odot 2019$ by author(s) and Scientific Research Publishing Inc. This work is licensed under the Creative Commons Attribution International License (CC BY 4.0).

http://creativecommons.org/licenses/by/4.0/

\begin{abstract}
The paper shows that the second law of thermodynamics and Pauli principle are implications of the Bell inequality.
\end{abstract}

\section{Keywords}

Thermodynamics, Quantum Theory, Relativity, Bell Inequality

\section{Introduction}

In a previous paper, [1] has been inferred two equations

$$
S=-\sum_{j} \Pi_{j} \log \Pi_{j}, \quad m_{r}=m \sqrt{1-v^{2} / c^{2}}, \quad 0 \leq v \leq c, \quad m_{r} \leq m,
$$

being $S$ the statistical definition of entropy of a system of particles, $\Pi_{j}$ the thermodynamic probability of the $j$-th state of the system and $v$ the modulus of velocity of one free particle having rest mass $m_{r}$ and dynamical mass $m$. According to these equations, trivial manipulations yield

$$
S^{o}=-\sum_{j} \Pi_{j} \frac{\delta S_{j}}{\delta \Pi_{j}}, \quad \Theta=\sum_{j} \Pi_{j},
$$

that imply

$$
\left(\frac{m_{r}}{m}\right)^{2}+\left(\frac{v}{c}\right)^{2}=1, \quad \frac{S}{\Theta}+\frac{S^{o}}{\Theta}=1, \quad 0 \leq \frac{S}{\Theta} \leq 1, \quad 0 \leq \frac{S^{o}}{\Theta} \leq 1, \quad \Theta=\sum_{j} \Pi_{j} .
$$

All equations reported here have been inferred in the conceptual frame of a unique theoretical model and contextually checked evidencing their sensible implications in the quoted paper. The first (1.3) was obtained examining the chance of energy fluctuation of a quantum particle related to the mass increase $m>m_{r}$ and evidenced the dual wave/corpuscle nature of matter. The addends ${ }^{\star}$ Retired physicist. 
concern indeed the probability of either behavior under appropriate experimental conditions. The second (1.3) outlines the order/disorder probabilities in a system of particles as a function of its allowed $j$ states. For the shortness, both (1.3) are justified here even without repeating the arguments exposed in the previous paper. The definition of $S^{o}$ is immediate consequence of that of $S$ : indeed $\delta S_{j} / \delta \Pi_{j}=-\log \Pi_{j}-1$, whence $-\Pi_{j} \delta S_{j} / \delta \Pi_{j}=S_{j}^{o}=-S_{j}+\Pi_{j}$ and thus $S^{o}$ summing over $j$. The second (1.3) follows thus by consequence. The first (1.3) is obtained simply squaring the second (1.1) that actually emphasizes the well accepted relativistic dependence of mass on velocity, previously inferred as a corollary.

Despite apparently nothing links (1.1), formally dissimilar and at first sight physically unrelated, it is however unquestionable the fact that

$$
\left(\frac{m_{r}}{m}\right)^{2}+\left(\frac{v}{c}\right)^{2}=\frac{S}{\Theta}+\frac{S^{o}}{\Theta}
$$

Also, the formal analogy between the Equations (1.3) suggests in particular the possible correspondences

$$
\left(\frac{m_{r}}{m}\right)^{2} \leftrightarrow \frac{S}{\Theta}, \quad\left(\frac{v}{c}\right)^{2} \leftrightarrow \frac{S^{o}}{\Theta}
$$

under the conditions (1.3) implied by (1.1); i.e.

$$
\sum_{j} \Pi_{j}>-\sum_{j} \Pi_{j} \log \Pi_{j}, \quad \sum_{j} \Pi_{j}>-\sum_{j} \Pi_{j} \frac{\delta S_{j}}{\delta \Pi_{j}} .
$$

Equation (1.3) is separately reasonable; as such, their worth is based on the chance of being contextually deductible along with other physical laws simply via the "ab initio" heuristic model [1]. It could seem weird, however, the further step of merging them in (1.4) and next splitting again this latter as in (1.5).

On the one hand, the mere numerical correspondence $a+b=1 \leftrightarrow a^{\prime}+b^{\prime}=1$ does not legitimate "per se" the physical meaning of the merged equation $a+b=a^{\prime}+b^{\prime}$ as done in (1.4); in other words it is necessary to demonstrate that the chance of inferring (1.4) from (1.3) to obtain (1.5) is sensible and implies sensible consequences, too. If so, then it is possible to replace $\leftrightarrow$ with the equality sign and define thereafter new statistical equations.

On the other hand, the Equation (1.4) is quite peculiar also for another reason; it equates the probabilistic behavior of one corpuscle/wave particle described by its ratios $m_{r} / m$ and $v / c$ to the statistical behavior of states allowed to a set of particles described by the sums (1.2) and (1.1).

However, since anyway (1.4) is formally legitimate, a form of correlation between the quantities (1.3) cannot be in principle excluded.

To introduce the statistical model proposed in this paper, regard first of all the modulus of velocity $v$ as the modulus of group velocity $v_{g}$ of a wave packet representing the De Broglie momentum of a given particle in the set. Regard next this $v_{g}$ as the average value $\left\langle v_{g}\right\rangle$ resulting from the various group 
velocities $v_{g j}$ of the set of particles constituting the system, as if in fact $\left\langle v_{g}\right\rangle$ would be itself one among the possible values of $v_{g i}$ : in a statistical set of states with corresponding $v_{g j}$, it is probable that $\left\langle v_{g}\right\rangle$ is very close to or even coincides with one among the allowed $v_{g j}$. So this $\left\langle v_{g}\right\rangle$ summarizing all $v_{g j}$ of the respective (1.1) and (1.2) describing the actual microstates of the system, can be related to (1.4) via the positions (1.3).

Trust therefore that a unique $\left\langle v_{g}\right\rangle$ appropriately defined, i.e. taking into account even the possible interactions between particles, is representative of all microstates of the system and compliant with (1.4): then it is in principle possible that both correspondences (1.5) have actual physical meaning, in which case sensible implications should be hidden in and deductible from them.

All considerations exposed in the following sections aim to clarify these points. Purpose of the paper is to show that the thermodynamic functions can be inferred directly from quantum first principles that concurrently imply relativistic outcomes as well.

The model highlights a further series of evidences additional to that carried outed in [1] to show the actual reasonableness of (1.5): this is the new contribution of this paper with respect to the previous one. The following considerations aim also to highlight the physical essence of these implications. With this premise, after a short remind of elementary statistical concepts in Section 2, the positions (1.5) are first concerned in the subsections 3 and 4 in a mere formal way through simple algebraic steps to infer preliminary corollaries, whose physical meaning is next exposed in the Sections 5 and 6. In particular the sections 3 to 5 are not a superfluous list of results already known; considering that (1.4) merges dual corpuscle/wave behavior of matter and entropy through their probabilistic meaning via the relativistic (1.1), the systematic check of both (1.5) is actually required to highlight their validity through new corollaries/implications exposed in the next Sections 7 and 8 . The results are proposed step by step to emphasize the underlying strategy: to link information on the microstates defining the terms of the sums (1.2) and (1.1) to the respective macrostates concerned by (1.3). The present paper completes [1] looking for further concepts already known while obtaining contextually also unprecedented results. The text is organized in order to be as self-contained as possible.

\section{Mathematical Tools}

The quantum uncertainty equations read

$$
\begin{gathered}
\delta x \delta p=n \hbar=\delta \varepsilon \delta t \\
\uparrow \\
n_{x}^{*} n_{p}^{*}=n=n_{\varepsilon}^{*} n_{t}^{*}
\end{gathered}
$$

inferred themselves as corollaries in [1]. The stars label arbitrary rational numbers expressing the range sizes as $n^{*}$ times the respective Planck units, $n$ is an arbitrary integer. 
It is essential to emphasize that by definition of quantum uncertainty all range sizes are unknown and conceptually unknowable; thus all $n^{*}$ are arbitrary and unspecifiable pure numbers. So, since even $n$ symbolizes an integer neither identified nor identifiable, it follows that both $\delta x \delta p$ and $\delta \varepsilon \delta t$ waive a specified reference system, inertial or non-inertial; although any $\delta x^{\prime} \delta p^{\prime} / \hbar$ implies its own $n^{\prime}$, in fact $n$ and $n^{\prime}$ are indistinguishable because actually both symbolize $n=1,2, \cdots$ and $n^{\prime}=1,2, \cdots$ identically. Thus the respective reference systems are in fact indistinguishable themselves as well. This conclusion is self-evident in the second dimensionless form (2.1).

Also note that, being in general $\Theta \neq 1$, the various $\Pi_{j}$ are thermodynamic probabilities, not mathematical probabilities with sum over all allowed $j$ normalized to the unity; instead by definition

$$
\mathcal{P}_{j}=\frac{\Pi_{j}}{\Theta}=\frac{\Pi_{j}}{\sum_{j} \Pi_{j}}
$$

is the mathematical probability of the $j$-th state. In general $v_{g}$ has statistical character: being related to $S^{o}$ and thus to $S$, it represents the average group velocity of the De Broglie momentum wave packets summarizing all states of the particles constituting the system, e.g. atoms or ions in a gas or a solid lattice. Is interesting in particular the average momentum wave group velocity $v_{g} \equiv\left\langle v_{g}\right\rangle$ related to and representative of all De Broglie matter waves allowed in the system. Introduce thus explicitly the notation $\left\langle v_{g}\right\rangle$ to emphasize that in (1.5) $v_{g}$ is actually to be regarded as mean value of all group velocities characterizing all states of the system. So, being the summations (1.6) compliant with and contributing to $\left\langle v_{g}\right\rangle$, write accordingly

$$
v \rightarrow v_{g} \rightarrow\left\langle v_{g}\right\rangle=\sum_{j} \frac{\Pi_{j}}{\Theta} v_{g j},
$$

where $v_{g j}$ is the $j$-th group velocity of the wave packet related to the respective state of the system concurring to define $S$ and $\delta S$. Also, analogous considerations hold for

$$
v^{2} \rightarrow v_{g}^{2} \rightarrow\left\langle v_{g}^{2}\right\rangle=\sum_{j} \frac{\Pi_{j}}{\Theta} v_{g j}^{2},
$$

e.g. useful to calculate the average kinetic energy $\left\langle\varepsilon_{g}\right\rangle$ of all $\varepsilon_{g j} \approx m v_{g j}^{2} / 2$ of the $j$-th wave packets.

To generalize these equations and calculate the average of any physical property, introduce an exemplificative function $f$ defined by

$$
f \rightarrow\langle f\rangle=\sum_{j} \frac{\Pi_{j}}{\Theta} f_{j} \equiv \frac{\sum_{j} f_{j}}{N},
$$

being $N$ the number of terms of summation, i.e. the number of allowed states. As done for $v_{g j}$, express the macroscopic average properties as summation over the aforesaid $j$ of local corresponding properties $f_{j}$. For example to find the sought 
statistical link between micro and macro states of $S,(1.1)$ yields in particular

$$
\begin{aligned}
& \langle S\rangle=-\Theta \sum_{j} \frac{\Pi_{j}}{\Theta} \log \Pi_{j}=-\Theta\langle\log \Pi\rangle=-\Theta\left\langle\log \frac{\Pi}{\Theta}\right\rangle+\text { const, } \\
& \text { const }=-\Theta \log \Theta,
\end{aligned}
$$

where const is a quantity not dependent on the summation index $j$. Here appears the average normalized probability $\Pi / \Theta$ plus a constant. So $\langle S\rangle / \Theta$ is the dimensionless Boltzmann entropy, usually expressed with positive sign as $S_{B}=\log W$ being $W=\Theta / \Pi$.

Moreover hold for the variation range $\delta f$ the useful statistical equations [2]

$$
\left\langle(\delta f)^{2}\right\rangle=\left\langle f^{2}\right\rangle-\langle f\rangle^{2},\left\langle f_{1} \cdot f_{2}\right\rangle=\left\langle f_{1}\right\rangle \cdot\left\langle f_{2}\right\rangle ;
$$

the left hand side has the meaning of mean square fluctuation of the statistical function $f$. Also, $f_{1}$ and $f_{2}$ are two physical properties related to the respective physical subsistems of that defining $f$; for example, as in the present context $S$ and $S^{o}$ define the disorder and order degree of a heterogeneous physical system, the subsistems described by $f_{1}$ and $f_{2}$ are two different volumes disordered and ordered of a whole crystal lattice. Particularly interesting is the first equation, rewritten as

$$
\frac{\left\langle(\delta f)^{2}\right\rangle}{\left\langle f^{2}\right\rangle}=1-\frac{v_{0}^{2}\langle f\rangle^{2}}{v_{0}^{2}\left\langle f^{2}\right\rangle}=1-\frac{\left\langle v^{2}\right\rangle^{2}}{\left\langle c^{2}\right\rangle}
$$

where the factor $v_{0}^{2}$ is here an arbitrary square velocity: taking advantage of the inequality $\left\langle f^{2}\right\rangle \geq\langle f\rangle^{2}$, easily verifiable in general assigning arbitrary values to the function $f$ and carrying out trivial calculations, it is possible to define $v_{0}$ in order that if $v_{0}^{2}\left\langle f^{2}\right\rangle=c^{2}$ then necessarily $v_{0}^{2}\langle f\rangle=v^{2}$. Thus (2.8) reads

$$
\frac{\left\langle(\delta f)^{2}\right\rangle}{c^{2} / v_{0}^{2}}=1-\frac{\langle v\rangle^{2}}{c^{2}}, \quad v_{0}^{2}\left\langle f^{2}\right\rangle=c^{2}, \quad v_{0}^{2}\langle f\rangle^{2}=\langle v\rangle^{2} \text {. }
$$

Hence

$$
\frac{\left\langle(\delta f)^{2}\right\rangle}{c^{2} / v_{0}^{2}}=\frac{c^{2}-\langle v\rangle^{2}}{c^{2}}=\frac{c^{2} \delta t^{2}-\langle v\rangle^{2} \delta t^{2}}{c^{2} \delta t^{2}}
$$

i.e.

$$
\left\langle(\delta f)^{2}\right\rangle=\frac{c^{2} \delta t^{2}-\left\langle\delta s_{g}\right\rangle^{2}}{\left(v_{0}^{2} / c^{2}\right) \delta s_{c}^{2}}=\frac{\delta \ell^{2}}{\left(v_{0}^{2} / c^{2}\right) \delta s_{c}^{2}}, \quad\left\langle\delta s_{g}\right\rangle=\left\langle v_{g}\right\rangle \delta t, \quad \delta s_{c}=c \delta t
$$

appears here the invariant interval $\delta \ell^{2}=c^{2} \delta t^{2}-\left\langle\delta s_{g}\right\rangle^{2}$ of the special relativity and the square length $\delta s_{c}^{2}$. To understand what has to do all of this with the entropy, note preliminarily that regarding $v$ as $v_{g}$ and thus $\left\langle v_{g}\right\rangle$ according to the previous considerations (2.3) and (2.4), the Lorentz factor (2.8) and the invariant interval (2.9) take statistical meaning, as they represent through $\left\langle v_{g}\right\rangle$ all $j$-th terms pertinent to the respective quantities defined by their own $v_{g j}$. This agrees with (1.3) and (1.5), because writing 


$$
\frac{v_{g}}{c}=\sqrt{\frac{S^{o}}{\Theta}}=\sqrt{1-\frac{S}{\Theta}}
$$

one infers that if $S$ has statistical meaning, then the same must hold also for $v_{g} / c$; in effect it will be also shown in the next Equation (3.3). This conclusion and the meaning of the factor $v_{0}$ are further concerned soon below.

Although reasonable, these preliminary remarks and that of section 1 are examined in the following three introductory sections. To carry out the next considerations are enough simply the average definitions of thermodynamic functions; in particular $\left\langle v_{g}\right\rangle$ is enough for the present purposes whatever the statistical distribution law of group velocities around this mean value might be.

\section{Preliminary Corollaries of (1.5)}

Relevant implications of both (1.5) are immediately recognizable writing formally

$$
\frac{S^{o}}{\Theta}=\frac{v_{g}^{2}}{c^{2}}=v_{g} \frac{m v_{g} / \gamma}{m c^{2} / \gamma}=\frac{p_{g} v_{g}}{\varepsilon_{g}}, \quad p_{g}=\frac{m v_{g}}{\gamma}, \quad \varepsilon_{g}=\frac{m c^{2}}{\gamma}
$$

the proportionality factor $\gamma$ has been purposely introduced for sake of generality. Thus, whatever $\gamma$ might actually be, it must be also true that

$$
p_{g}=\frac{v_{g} \varepsilon_{g}}{c^{2}}
$$

as it is immediate to verify.

First of all, quoting shortly [1], (3.2) yields $\delta\left(v_{g j} / c\right)=\delta\left(p_{g j} c / \varepsilon_{g j}\right)$ and thus

$$
\begin{aligned}
\delta\left(\frac{v_{g j}}{c}\right) & =\frac{p_{g j} c}{\varepsilon_{g j}}\left(\frac{\delta\left(p_{g j} c\right)}{p_{g j} c}-\frac{\delta \varepsilon_{g j}}{\varepsilon_{g j}}\right)=\frac{p_{g j} c}{\varepsilon_{g j}} \delta \log \left(\frac{p_{g j} c}{\varepsilon_{g j}}\right) \\
& =\frac{p_{g j} c}{\varepsilon_{g j}} \log \left(\frac{p_{g j} c}{\varepsilon_{g j}}\right)-\frac{p_{g j} c}{\varepsilon_{g j}} \log \left(\frac{p_{0} c}{\varepsilon_{0}}\right) ;
\end{aligned}
$$

the last step has implemented explicitly the general definition of range $\delta(p / \varepsilon)=p / \varepsilon-p_{0} / \varepsilon_{0}$. So, defining

$$
\Pi_{j}=\frac{v_{g j}}{c}<1, \quad W_{j}=\frac{p_{g j} c}{\varepsilon_{g j}}<1, \quad W_{0}=\frac{p_{0} c}{\varepsilon_{0}},
$$

(3.3) implies $\delta \Pi_{j}=W_{j} \log W_{j}-W_{j} W_{0}$; thus, calling by definition $\delta \Pi_{j}=\Pi_{j}-\Pi_{v j}$, summing over $j$ one finds

$$
\langle\delta \Pi\rangle=\langle\Pi\rangle-\left\langle\Pi_{v}\right\rangle=\sum_{j} W_{j} \log W_{j}-\langle W\rangle W_{0}
$$

being thus reasonably

$$
\left\langle\Pi_{v}\right\rangle=-\sum_{j} W_{j} \log W_{j}, \quad\langle\Pi\rangle=-\langle W\rangle W_{0} .
$$

Even the average group velocity probability $\left\langle\Pi_{v}\right\rangle$ fulfills an entropy-like law; indeed, as it is possible to put purposely the arbitrary constants $p_{0}$ and $\varepsilon_{0}$ 
such that $p_{0} c=\varepsilon_{0}$, results in fact $W_{0}=0$.

Moreover, combining (3.1) squared and (3.2) one finds

$$
\varepsilon_{g}^{2}-\left(p_{g} c\right)^{2}=\varepsilon_{g}^{2}\left(1-\frac{v_{g}^{2}}{c^{2}}\right)=\frac{\left(m c^{2}\right)^{2}}{\gamma^{2}}\left(1-\frac{v_{g}^{2}}{c^{2}}\right),
$$

whence the reasonable correspondences

$$
\varepsilon_{g}^{2}-\left(p_{g} c\right)^{2} \leftrightarrow\left(m c^{2}\right)^{2}, \quad \gamma^{2} \leftrightarrow 1-\frac{v_{g}^{2}}{c^{2}}
$$

and thus

$$
\varepsilon_{g}^{2}-\left(p_{g} c\right)^{2}=\left(m c^{2}\right)^{2}, \quad \gamma^{2}=1-\frac{v_{g}^{2}}{c^{2}} .
$$

It is worth emphasizing that the last two (3.1) and then (3.2) along with (3.6) have been obtained merely via the second (1.5) only, without additional hypotheses. So the equalities (3.1) yield themselves self-consistently the correct relativistic momentum and energy of the free particle, which in turn takes statistical meaning through the aforesaid steps $v \rightarrow v_{g} \rightarrow\left\langle v_{g}\right\rangle:$ it is enough to express (3.1) as a function of $\left\langle v_{g}\right\rangle$ instead of $v$. Eventually the first (1.5) yields according to (1.3)

$$
\frac{S}{\Theta}=\frac{c^{2}-v_{g}^{2}}{c^{2}}=\frac{c^{2} \delta t^{2}-\delta s_{g}^{2}}{c^{2} \delta t^{2}}, \quad \delta s_{g}^{2}=v_{g}^{2} \delta t^{2},
$$

whence the possible correlation chances

$$
S \leftrightarrow \delta \ell^{2}, \quad \Theta \leftrightarrow \delta s_{c}^{2}, \quad \delta \ell^{2}=c^{2} \delta t^{2}-\delta s_{g}^{2}, \quad \delta s_{c}=c \delta t
$$

that agree with (2.9) and imply with the help of (1.1) and first (1.5)

$$
\frac{S}{\Theta}=\frac{\delta \ell^{2}}{\delta s_{c}^{2}}=\left(\frac{m_{r}}{m}\right)^{2}=\gamma^{2} .
$$

If so, then $S$ should be a relativistic invariant. In effect, this conclusion agrees with that early inferred by Planck [3], who concluded that "the entropy of the body does not depend on the choice of the reference frame"; i.e. the disorder degree of a body appears the same to two inertial observers in reciprocal motion. Consider now the first equality (3.8)

$$
\frac{S}{\Theta / \gamma^{2}}=\frac{\delta \ell^{2}}{\delta s_{c}^{2} / \gamma^{2}}
$$

suggests that $\Theta$ defined in $R$ implies regarding $\delta s_{g} / \gamma$ as $\delta s_{g}^{\prime}$ in another $R^{\prime}$; indeed in $R^{\prime}$ it is possible to write

$$
\frac{S}{\Theta^{\prime}}=\frac{\delta \ell^{2}}{\delta s_{c}^{\prime 2}}, \quad \Theta^{\prime}=\Theta / \gamma^{2} \leftrightarrow \delta s_{c}^{\prime 2}=\delta s_{c}^{2} / \gamma^{2} .
$$

In other words $\Theta$ defined as a square range size transforms like a square time length inherent the definition of $\delta s_{c}$ : as it is reasonable to expect, the Lorentz time dilation affects all probabilities $\Pi_{j}$ allowed to the system of 
particles and thus the resulting $\Theta$.

On the one hand is evident the connection of (3.9) with (2.9), since both have the same form if $v_{0}^{2} / c^{2}=\gamma^{2}$; then

$$
v_{0}^{2}=c^{2}-v_{g}^{2}, \quad \frac{S}{\Theta \gamma^{2}}=\left\langle(\delta f)^{2}\right\rangle .
$$

So $S / \Theta$ appears as mean square fluctuation of an appropriate function $f$ defining $v_{g}^{2} / c^{2}$ in agreement with (1.4) and (1.5), whereas it appears that

$$
\left(v_{0} / c\right)^{2}+\left(\left\langle v_{g}\right\rangle / c\right)^{2}=1 \text {. }
$$

On the other hand further information is provided by (3.10) itself noting that in general $c \gamma^{2}=v_{g}^{*}$, being thus $v_{g}^{*}$ any group velocity different from $c$. Thus the last (3.7) implies the following chains of equations

$$
\Theta \gamma^{2}=c^{2}(\gamma \delta t)^{2}=(c \delta t) \delta s_{c}^{*}=c\left(\frac{\delta t}{\gamma}\right)\left(\gamma \delta s_{c}^{*}\right), \quad \delta s_{c}^{*}=v_{g}^{*} \delta t
$$

whence

$$
\left(\frac{\delta t}{\gamma}\right)\left(\gamma \delta s_{c}^{*}\right)=\delta s_{c}^{*} \delta t=\delta s_{c}^{s^{*}} \delta t^{\prime}, \quad \delta s_{c}^{\prime^{*}}=\gamma \delta s_{c}^{*}, \quad \delta t^{\prime}=\frac{\delta t}{\gamma}:
$$

i.e. one finds the Lorentz space contraction $\delta s_{c}^{\prime^{*}}=\gamma \delta s_{c}^{*}$ and time dilation $\delta t^{\prime}=\delta t / \gamma$.

The connection of these considerations with the quantum theory is immediate with the help of (3.7) and (2.1), simply noting that (3.7) defines an invariant time

$$
\delta t_{i n v}= \pm \frac{\delta \ell}{c}= \pm \frac{c \delta t}{c} \sqrt{1-\frac{\delta s_{g}^{2} / \delta t^{2}}{c^{2}}}= \pm \delta t \sqrt{1-\frac{v_{g}^{2}}{c^{2}}}, \quad v_{g}^{2}=\frac{\delta s_{g}^{2}}{\delta t^{2}} .
$$

Thus, writing identically

$$
\frac{1}{\delta t}= \pm \frac{1}{\delta t_{i n v}} \sqrt{1-\frac{v_{g}^{2}}{c^{2}}}
$$

and multiplying both sides by $h$, one finds

$$
\delta \varepsilon= \pm \delta \varepsilon_{i n v} \sqrt{1-\frac{v_{g}^{2}}{c^{2}}}, \quad \delta \varepsilon=\frac{\hbar}{\delta t}, \quad \delta \varepsilon_{i n v}=\frac{\hbar}{\delta t_{i n v}} .
$$

On the one hand $\delta \varepsilon=\varepsilon-\varepsilon_{\ell}$; thus, being correspondingly $\delta \varepsilon_{i n v}=\varepsilon_{i n v}-\varepsilon_{0 i n v}$, the first equation splits as follows

$$
\varepsilon= \pm \varepsilon_{i n v} \sqrt{1-\frac{v_{g}^{2}}{c^{2}}}, \quad \varepsilon_{\ell}= \pm \varepsilon_{0 i n v} \sqrt{1-\frac{v_{g}^{2}}{c^{2}}}:
$$

i.e. putting $\varepsilon_{0 i n v}=m c^{2}$ the second equation yields the relativistic Lagrangian $\varepsilon_{\ell}$ of a free particle, whereas the first equation shows that $\varepsilon_{i n v}$ requires the Lorentz transformation rule of energy $\varepsilon \sim t$. Both boundaries of the energy range $\delta \varepsilon$ have thus their own physical meaning. Further considerations on these well known results are unnecessary and waived. 
On the other hand (2.1) yields also $\delta \varepsilon_{g}=n \hbar / \delta t$, i.e.

$$
\delta \varepsilon_{g}= \pm \frac{n \hbar \omega_{g}}{\sqrt{1-v_{g}^{2} / c^{2}}}, \quad \omega_{g}=\frac{2 \pi}{\delta t} ;
$$

of course $\delta \varepsilon_{g}=\varepsilon_{g 2}-\varepsilon_{g 1}$ at the left hand side is the range size that encloses all energies $n \hbar \omega_{g}^{*}$ at the right hand side compatible with any arbitrary integer $n$. Hence to any particular $n^{*} \hbar \omega_{g}^{*}$ corresponds the value $\varepsilon_{g}^{*}$, i.e.

$$
\varepsilon_{g}^{*}= \pm \frac{n^{*} \hbar \omega_{g}^{*}}{\sqrt{1-v_{g}^{2} / c^{2}}}, \quad \varepsilon_{1 g} \leq \varepsilon_{g}^{*} \leq \varepsilon_{2 g} .
$$

Being the boundaries of $\delta \varepsilon$ arbitrary means that actually $n$ can take any integer value. A reasoning exactly similar implements the further chance of inferring from (GGG)

$$
\delta p_{x}= \pm \frac{n \hbar \kappa_{g}}{\sqrt{1-\frac{v_{g}^{2}}{c^{2}}}}, \quad \kappa_{g}=\frac{2 \pi}{\delta x_{g}},
$$

where $\kappa_{g}$ is actually the so called wave vector. Thus, as before,

$$
p_{x}^{*}= \pm \frac{n^{*} \hbar \kappa_{g}^{*}}{\sqrt{1-\frac{v_{g}^{2}}{c^{2}}}} .
$$

The connection with the relativistic (3.1) is of course given by the following specific correlations

$$
n^{*} \hbar \omega_{g}^{*} \rightarrow m c^{2}, \quad n^{*} \hbar \kappa_{g}^{*} \rightarrow m v_{g} .
$$

Note that the double sign of $p_{x}$ clearly corresponds to both $x$-components of momentum, that of the energy prospects the existence of negative states of energy. Also, all of this introduces the Planck energy $\hbar \omega_{g}$ and De Broglie momentum $h / \lambda$ corresponding to $\hbar \kappa_{g}$; obviously the Lorentz factor merges with their respective $t^{-1}$ and $\lambda^{-1}$ dependencies.

As a preliminary conclusion, therefore, the hints prospected in the present section by both (1.5) appear sensible enough and stimulating to deserve further investigation, in particular as concerns the direct link between thermodynamics and relativity. As the quantum definition (1.1) of entropy is already evident through its statistical meaning related to the probability of allowed states, actually this link is reasonably expected to highlight the connection between quantum probabilistic and relativistic theory. These non trivial conclusions justify the reasoning hitherto carried out via its possible implications.

Note eventually that $p_{g} v_{g}=\varepsilon_{g} v_{g}^{2} / c^{2}$ of (3.2) with $v_{g} \leq c$ implies itself

$$
\varepsilon_{g}-p_{g} v_{g} \geq 0
$$

this result indeed agrees with that inferable in an analogous way from (3.5), which implies it "a fortiori". As this inequality holds even multiplying both sides by an arbitrary coefficient $\theta>0$, it is possible to write the dimensionless 
inequality

$$
\frac{\varepsilon_{g}}{\theta}-\frac{p_{g} v_{g}}{\theta}=S_{\varepsilon}-S_{p v} \geq 0, \quad S_{\varepsilon}=\frac{\varepsilon_{g}}{\theta}, \quad S_{p}=\frac{p_{g} v_{g}}{\theta}
$$

where $\theta$ represents an arbitrary energy factor. (3.13) is significant because it implies (3.14) that defines $S$ as the ratio of two energies and evidences its fundamental property

$$
\varepsilon_{g}-p_{g} v_{g}=\theta \delta S_{\varepsilon p} \geq 0, \quad \delta S_{\varepsilon p}=S_{\varepsilon}-S_{p v} .
$$

In this respect, note that (3.14) is not "ad hoc" position: implementing once more (1.5), it is easy to acknowledge that $S$ defined by (3.14) is actually the same function introduced in (1.1). Indeed, proceeding in analogy with (3.1), one finds that just (1.5) allow writing

$$
\frac{S}{\Theta}=\frac{m_{r}}{m} \frac{m_{r}}{m}=\gamma \frac{m_{r}}{m}=\frac{m_{r} c^{2}}{m c^{2} / \gamma}=\frac{\varepsilon^{*}}{\varepsilon^{* *}}
$$

where $S$ is in fact an energy ratio; regarding of course $\Theta$ still as that introduced in (3.7), this suggests the correlations

$$
S \leftrightarrow \Theta \frac{\varepsilon^{*}}{\varepsilon^{* *}}, \quad \Theta \leftrightarrow \delta t^{2} .
$$

So, whatever $\varepsilon^{*}$ and $\varepsilon^{* *}$ might be, the proportionality constant $\theta$ justifies in principle the link between $S_{\varepsilon}$ and $S_{p v}$ of (3.14) to that hitherto concerned via (1.1) only; are crucial in this respect the positions (1.5).

As a closing remark, note that in general owing to (3.14) it is possible to expand in series (3.15) as

$$
\delta S_{\varepsilon p}=\left(m c^{2}-m v_{g}^{2}\right) \frac{1}{\sqrt{1-\frac{v_{g}^{2}}{c^{2}}}} \approx m c^{2}-1 / 2 m v_{g}^{2}+\cdots, \quad v_{g} \ll c ;
$$

recalling now (2.4) and (2.5), average both sides and implement $v_{g}^{2} \rightarrow\left\langle v_{g}^{2}\right\rangle$ to write thanks to (3.15)

$$
\left\langle\theta S_{\varepsilon}\right\rangle=\left\langle\varepsilon_{g}\right\rangle=m c^{2},\left\langle\theta S_{p v}\right\rangle \approx \frac{1}{2} m\left\langle v_{g}^{2}\right\rangle+\cdots
$$

The importance of this result and (3.15), better explained in the next section, appears even here regardless of any information or hypothesis about $\varepsilon_{g}$ and $p_{g} v_{g}$; it is simply due to $0 \leq v_{g} \leq c$ allowing (1.3) and (1.4). Precisely for this reason it holds for an isolated system not subjected to any external action that would necessarily require an appropriate external energy $\varepsilon_{\text {ext }}$ exchange to be introduced in the previous equations; this action could perturb and modify the spontaneous evolution of the system leading, as so far described, to (3.15).

In the present model, owing in particular to both positions (1.5), quantum, relativistic and thermodynamic results merge in a natural and elementary way.

The definition (3.8) of entropy as ratio of two square lengths is seemingly weird; actually however it is not so, the next section shows that this definition is closely related to two significant relativistic corollaries. 


\section{Black Hole Surface Entropy and Red Shift}

It is known that the Hawking surface entropy of a black hole is in fact the ratio of square lengths [4], like (3.8). So it is sensible to start just from this equation and write

$$
S=\Theta \frac{\delta \ell^{2}}{\delta s_{c}^{2}} \rightarrow S_{b h}=\chi \Theta_{b h} \frac{\ell_{b h}^{2}}{\ell_{b h}^{* 2}}, \quad \ell_{b h}=\frac{2 m G}{c^{2}}, \quad \ell_{b h}^{*}=\frac{2 m^{*} G}{c^{2}},
$$

being $\ell_{b h}$ the black hole radius of mass $m$ and $\chi$ an appropriate proportionality constant expressing $\Theta=\chi \Theta_{b h}$. The reason of these positions is intuitive: the general Equation (3.8) is here purposely specified to describe in particular the surface entropy of a black hole; thus the positions (4.1) are not "ad hoc" hypotheses, rather they fit the physical meaning of the actual problem concerned here. To this aim is necessary to regard $\delta \ell$ of (3.7) as length $\ell_{b h}$ consistent with $m$, whereas the proportionality constant $\chi$ can be exploited in order to define the surface of a sphere; accordingly $\delta s_{c}$ must turn into the new reference length $\ell_{b h}^{*}$, radius of a black hole of arbitrary mass $m^{*}$. In other words, $\delta s_{c} \rightarrow \delta \ell_{b h}^{*}$ is the new scale factor coherent with $\delta \ell \rightarrow \delta \ell_{b h}$ in defining the dimensionless $S \rightarrow S_{b h}$. In this respect is also appropriate the position

$$
S_{b h}=\frac{4 \pi \ell_{b h}^{2}}{\ell_{b h}^{* 2}}, \quad \chi=\frac{4 \pi}{\Theta_{b h}}
$$

this definition of $\chi$ makes the particular form of $\Theta_{b h}$ introduced in (4.1) compliant with the black hole surface $A_{b h}=4 \pi \ell_{b h}^{2}$ of radius $\ell_{b h}$ and given mass $m$, whereas $\delta s_{c}$ becomes the dimensional proportionality constant $\ell_{b h}^{*-2}$ between dimensionless surface entropy $S_{b h}$ and surface area $A_{b h}$. In this respect, a reasonable way to define uniquely $S_{b h}$ is to implement Planck units and thus to identify the constant $m^{*}$ with the Planck mass. Put then

$$
m^{* 2}=m_{p l}^{2}, \quad m_{p l}=\sqrt{\frac{\hbar c}{G}}, \quad \text { i.e. } \quad \ell_{b h}^{* 2}=\ell_{b h}^{* 2}\left(m^{*}=m_{p l}\right)=4 \ell_{p l}^{2}, \quad \ell_{p l}=\sqrt{\frac{\hbar G}{c^{2}}},
$$

so that $(4.2)$ reads

$$
S_{b h}=\frac{4 \pi \ell_{b h}^{2}}{4 \ell_{p l}^{2}}=\frac{A_{b h}}{4 \ell_{p l}^{2}}
$$

This is the well known Hawking-Beckenstein surface entropy of a black hole. Are omitted for brevity further considerations on this result.

It is worth remarking once more that the crucial point here is not the fact itself of having obtained this result, already known, but mostly to have confirmed once more (1.3) and the link (1.5) between the probabilistic corpuscle/wave behavior of matter and probabilistic concept of entropy appearing in the first (1.1). In particular is significant the fact of having found again a sensible result implementing $S / \Theta$ as weird ratio of square lengths.

Consider now that the Equation (3.7) allows defining an invariant time $\tau$ defined by 


$$
\tau^{2}=\frac{\delta \ell^{2}}{c^{2}}=\delta t^{2}-\frac{\delta s_{g}^{2}}{c^{2}},
$$

which can be rewritten via frequencies as follows with obvious meaning of symbols

$$
\frac{\delta s_{g}^{2}}{c^{2}}=\delta t^{2}-\frac{\delta \ell^{2}}{c^{2}}=\frac{1}{v^{2}}-\frac{1}{v_{\ell}^{2}}=\frac{v_{\ell}^{2}-v^{2}}{\left(v_{\ell} v\right)^{2}} ;
$$

thus, being by definition $\delta s_{g}$ the difference between two arbitrary space coordinates $s_{2}-s_{1}$, this result yields

$\frac{v_{\ell}^{2} \delta s_{g}^{2}}{c^{2}}=\frac{\delta \varphi}{c^{2}}=\frac{\delta v}{v^{2} /\left(v_{\ell}+v\right)}=\frac{\delta v}{v_{0}}, \quad v_{0}=\frac{v^{2}}{v_{\ell}+v}, \quad \delta v=v_{\ell}-v_{g}, \quad \delta \varphi=v_{\ell}^{2} \delta s^{2}$.

In the present context, regard once more $v \rightarrow v_{g} \rightarrow\left\langle v_{g}\right\rangle$ exactly as done in (2.3) and (2.5); in turn $\left\langle v_{g}\right\rangle$ results from all $v_{g j}$, whereas $\delta s_{g}^{2} \rightarrow\left\langle\delta s_{g j}^{2}\right\rangle$ with $\left\langle\delta s_{g j}^{2}\right\rangle$ resulting from all $\delta s_{g j}^{2}$. These positions allow regarding accordingly (4.3), i.e. with reference to its representation of each $j$-th state. Next, taking the average of both sides after summing over all states as done in (2.5), one finds with the help of (2.7) whatever $\delta v=v_{g}-v_{0 g}$ and $v_{0 g}$ might be

$$
\frac{\langle\delta \varphi\rangle}{c^{2}}=\left\langle\frac{\delta v}{v_{0 g}}\right\rangle=\frac{\langle\delta v\rangle}{\left\langle v_{0 g}\right\rangle}, \quad\langle\delta \varphi\rangle=v_{\ell}^{2} s_{2}^{2}-v_{\ell}^{2} s_{1}^{2}, \quad s_{1}^{2}=\left\langle s_{1 g}^{2}\right\rangle, \quad s_{2}^{2}=\left\langle s_{2 g}\right\rangle^{2} .
$$

The first equation introduces the average of a function $\delta \varphi$ having physical dimension of square velocity, to which corresponds an average $\delta v$; as before, $c^{2}$ and $\left\langle v_{0 g}\right\rangle$ are the respective reference values. These terms are easily recognizable multiplying both sides of $\varphi$ by an arbitrary mass $m$, which yields the dimensional relationship

$$
m \varphi=m(s v)^{2}=\text { energy : }
$$

this equation shows that if $\varphi=$ energy/mass is in particular the gravitational potential, then it also appears that the frequency shift $\left\langle\delta v / v_{0 g}\right\rangle$ is related to $\left\langle\delta \varphi / c^{2}\right\rangle$ [5]. It is worth noticing in this respect that (3.7) and (3.8), the key equations bringing to (4.3) and (4.4), are usually inferred as properties of the space time; in particular the identification of $\varphi$ with the gravitational potential guessed via mere dimensional basis, can be better understood showing that $\varphi$ is proportional to $S / \Theta$. Note indeed that the Equation (1.5) yields

$$
\frac{S}{\Theta}=\left(\frac{m_{r}}{m}\right)^{2}=\frac{m_{r}}{m} \gamma=\frac{m_{r}}{m} \frac{\ell_{r} \gamma}{\ell_{r}}=\frac{m_{r}}{m} \frac{\ell}{\ell_{r}},
$$

where $\ell_{r}$ is an arbitrary length in the reference system $R$ were is defined the rest mass $m_{r}$; in the last step $\ell=\ell_{r} \gamma$ is the Lorentz contraction of $\ell_{r}$ in another inertial reference system $R^{\prime}$ moving with respect to $R$ at arbitrary rate $v$ corresponding to that defined by $\gamma$. So to show that

$$
\varphi \propto \frac{(m S) / m_{r}}{(\ell \Theta) / \ell_{r}},
$$


let us regard the rest mass and length as constants; as previously done to infer (WKQ) from (4.1), $m_{r}$ and $\ell_{r}$ fix the scale of the $\gamma$ dependent length $\ell$ and mass $m$ in $R^{\prime}$. Let for example $c^{2}$ the proportionality constant consistently with the physical dimensions of $\varphi$; including then $m_{r}$ and $\ell_{r}$ in this proportionality constant, write

$$
\varphi=G \frac{m S}{\ell \Theta}, \quad G=c^{2} \frac{\ell_{r}}{m_{r}},
$$

being $G$ the aforesaid proportionality constant; so in the particular case of an attractive field

$$
\varphi=-\frac{G m_{e f f}}{\ell_{e f f}}, \quad m_{e f f}=m S, \quad \ell_{e f f}=\ell \Theta:
$$

The notation emphasizes that $m_{\text {eff }}$ is the effective dynamical mass $S m$ in $m_{r}$ units modulated by $S$. Consider for example an atom in an amorphous solid or in a crystal lattice: the dynamical mass $m$ is related to its rest mass $m_{r}$ not only via the Lorentz factor, see (1.1), but also through the degree of order/disorder dimensionless factor $S$ characterizing the lattice interaction. In other words $m_{\text {eff }}$ accounts for the way the motion of the atom is perturbed by the neighbors in a given kind of lattice. Analogous reasoning holds for $\ell_{\text {eff }}$. Anyway the definitions of effective mass and length highlight the expected form of the gravitational potential; owing to the choice of $G$ as proportionality constant, $\varphi$ has in effect physical dimensions of square velocity, as indeed it appears in (4.4).

Is interesting the fact that the function $\langle\varphi\rangle$ results calculated in (4.4) at two different points $s_{1}$ and $s_{2}$ where are defined the respective averages, whereas the boundaries of the frequency range size $\langle\delta v\rangle$ are related to $\langle\delta \varphi\rangle$. This is the statistical formulation of the red shift $\langle\delta v\rangle$ of a beam of photons having average frequency $\left\langle v_{0 g}\right\rangle$ moving radially from or towards the gravity center by effect of an average gravitational potential difference $\langle\delta \varphi\rangle$, while instead the deterministic approach of general relativity yields the frequency shift of one photon traveling between the local points $s_{1}$ and $s_{2}$ in the presence of a central gravity field; this information is replaced here by a statistical reasoning leading to the same conclusion, now however probabilistic and non deterministic thus fully compatible with the ideas of quantum theory.

\section{Thermodynamic Corollaries}

All considerations of the previous section did not need introducing explicitly the microstates appearing at the right hand sides of (1.2); this is done now. As a preliminary consideration note that owing to (1.3)

$$
\frac{\delta S_{j}}{\delta \Pi_{j}} \leq 0,
$$

which implies that $\delta S_{j}$ increases when decreases the range size allowed to the various $\Pi_{j}$ of the states of the system in order that $S_{j}^{o}$ are positive. Implement the first equality (3.1) according to (3.2) 


$$
\frac{S^{o}}{\Theta}=\frac{p_{g} v_{g}}{\varepsilon_{g}}=-\sum_{j} \frac{\Pi_{j}}{\Theta} \frac{\delta S_{j}}{\delta \Pi_{j}}=-\sum_{j} \frac{\delta S_{j}}{\delta \log \Pi_{j}},
$$

through which is easily proven the aforesaid statistical meaning of $\varepsilon_{g}$ and $p_{g}$ on the one hand and $v_{g}$ on the other hand, see (3.3) and (3.4); owing to (2.5), it is possible to regard now also (3.2) as

$$
\frac{p_{g} v_{g}}{\varepsilon_{g}} \rightarrow\left\langle\frac{p_{g} v_{g}}{\varepsilon_{g}}\right\rangle
$$

in order to obtain from (5.2)

$$
\Theta\left\langle\frac{p_{g} v_{g}}{\varepsilon_{g}}\right\rangle=\Theta \frac{\left\langle v_{g}^{2}\right\rangle}{c^{2}}=\Theta \sum_{j} \frac{\Pi_{j}}{\Theta} \frac{p_{g j} v_{g j}}{\varepsilon_{g j}}=-\sum_{j} \Pi_{j} \frac{\delta S_{j}}{\delta \Pi_{j}} .
$$

In agreement with (5.1), Equation (5.4) is fulfilled by

$$
\frac{p_{g j} v_{g j}}{\varepsilon_{g j}}+\frac{\theta \delta S_{j}}{\theta \delta \Pi_{j}}=0
$$

where $\theta$ formally introduced in (3.14) is an arbitrary function to be defined requiring that it does not depend explicitly on the index $j$. The validity of the steps (5.3) to (5.5) is supported examining their implications. The fact of introducing the explicit definitions of momentum $p_{g j}$ and energy $\varepsilon_{g j}$ of the $j$-th matter wave packet along with dimensionless $\delta S_{j}$, suggests a general way to link side by side numerator and denominator of (5.5) for any $j$-th index just through $\theta$, i.e.

$$
p_{g j} v_{g j} \leftrightarrow \theta \delta S_{j}, \quad \varepsilon_{g j} \leftrightarrow-\theta \delta \Pi_{j}, \quad \delta S_{j}=S_{j}-S_{0 j}, \quad \delta \Pi_{j}=\Pi_{j}-\Pi_{0 j} ;
$$

in this way $\theta$ appears again as an energy factor. The minus sign depends on how is defined $\delta \Pi_{j}$ in (5.1): clearly $S_{0 j}$ and $\Pi_{0_{j}}$ are arbitrary values expressing explicitly the boundaries of the respective ranges. In effect, whatever the $j$ indexed and non-indexed quantities might be, (5.5) is verified dividing side by side and then summing the first two (5.6). Combining $p_{g j} v_{g j}=\theta \delta S_{j}$ and $\varepsilon_{g j}=-\theta \delta \Pi_{j}$ the single $j$-th terms (5.6) and performing their summations, are obtained macrscopic functions $\delta S$ and $U$ defined as follows

$$
\begin{aligned}
& \theta \delta S_{j}=p_{g j} v_{g j} \rightarrow \theta \delta S=\theta \sum_{j} \delta S_{j}=\sum_{j} p_{g j} v_{g j} \\
& \varepsilon_{g j}=-\theta \delta \Pi_{j} \rightarrow U=\sum_{j} \varepsilon_{g j}=-\theta \sum_{j} \delta \Pi_{j} .
\end{aligned}
$$

Subtracting side by side one finds

$$
U-\theta \delta S=\mathcal{E}, \quad U=-\theta \delta \Theta, \quad \mathcal{E}=\sum_{j}\left(\varepsilon_{g j}-p_{g j} v_{g j}\right) \geq 0,
$$

where $\mathcal{E}$ and $U$ are energies and

$$
\theta \delta S=\sum_{j} p_{g j} v_{g j}=\mathcal{Q} \text { i.e. } \quad \delta S=S-S_{0}=\frac{\mathcal{Q}}{\theta} .
$$

To explain these equations, write explicitly the ranges 


$$
\theta \delta S=\theta\left(S-S_{0}\right), \quad \theta \delta \Theta=\theta\left(\Theta-\Theta_{0}\right), \quad \Theta_{0}=\sum_{j} \Pi_{0 j}
$$

and next expand and recombine conveniently these addends appearing in (5.8); the second and first terms read respectively

$$
U+\theta \Theta=\theta \Theta_{0}, \quad \mathcal{E}=-\theta \Theta+\theta \Theta_{0}-\theta S+\theta S_{0} \geq 0 .
$$

Then calling

$$
\theta \Theta_{0}=H, \quad \theta \Theta=L, \quad \theta S_{0}=\mathcal{L},
$$

the Equation (5.10) read

$$
H=U+L, \quad \mathcal{E}=U+\mathcal{L}-\theta S \geq 0 .
$$

Clearly, putting $\Theta=S_{0}$ in (5.10), (5.12) with $\mathcal{L}=L$ reads $\mathcal{E}=H-\theta S$; the notation $\mathcal{L} \neq L$ has been proposed for sake of generality. The positions (5.11) make consistent the definition of $\mathcal{E}$ in (5.10) and (5.12): $\mathcal{E}$ is the Gibbs free energy, whereas with $\theta S_{0}=\mathcal{L}=0$ one recognizes the Helmholtz free energy. Eventually to clarify the meaning of $\mathcal{L}$ write now

$$
\theta \delta S=\delta U+\delta \mathcal{L} \geq 0,
$$

being $\delta \mathcal{L}$ an amount of energy balancing $\delta U$ and $\theta \delta S$. The $\geq L$ notation is suggested by (3.15) and confirmed more in general soon below.

On the one hand, owing to (5.9) and (5.13),

$$
\mathcal{Q}=\delta U+\delta \mathcal{L} .
$$

On the other hand

$$
\delta(\theta S)-S \delta \theta=\delta U+\delta \mathcal{L}
$$

yields

$$
-S \delta \theta=\delta(U+\mathcal{L}-\theta S)=\delta E:
$$

so, thanks to the position (5.13) appears at the right hand side the same function $\mathcal{E}$ defined in (5.12). Specify now for example $\mathcal{L}=P V$, where $P$ and $V$ are external pressure acting on the system and volume of the system, so that $\delta \mathcal{L}=P \delta V+V \delta P$; then at constant $P$ one finds

$$
-S=\frac{\delta(U+P V-\theta S)}{\delta \theta} .
$$

The last equation at constant $P$ is the well known thermodynamic definition of entropy, whereas (5.14) is the first law taking $\mathcal{Q}$ as heat affecting internal energy change $\delta U$ and work $\delta \mathcal{L}$ done on/from the system.

To clarify the link between $\mathcal{L}$ and $L$ put $\mathcal{L}=L+X$, being $X$ a positive or negative quantity to be specified. So in (5.10) $\mathcal{E}=U+L+X-\theta S \geq 0$, i.e. $\mathcal{E}-H=X-\theta S \geq 0$. The right hand side is consistent however with $X \geq 0$ only, so that $\mathcal{L} \geq L$. Thus with $\delta \mathcal{L} \geq \delta L$ (5.13) reads $\mathcal{Q} \geq \delta U+\delta L$, i.e. exists an amount $\delta Q$ such that $\mathcal{Q} \geq \delta Q$ and $\delta Q=\delta U+\delta L$. Hence (5.9) and $\mathcal{Q} \geq \delta Q$ merge first and second law writing (5.14) simply as

$$
\theta \delta S \geq \delta Q=\delta U+\delta L .
$$


This result is the first law and the property of $S$ of being equal to $\delta Q / \theta$ for reversible process and $>\delta Q / \theta$ for irreversible processes; also, it clarifies the link between $L$ and $\mathcal{L} \geq L$.

Further comments on these results are superfluous; the fact of having introduced the factor $\theta$ in (3.14) and (5.5) is thus not merely formal.

Two significant implications of these results allow to highlight more clearly the thermodynamic meaning of (3.15).

1) The second line of Equation (5.7) reads $\varepsilon_{g j}=-\theta \Pi_{j}+\theta \Pi_{0 j}$, which introduces

$$
\theta \Pi_{j}=\theta \Pi_{0 j}-\varepsilon_{g j}=\delta \epsilon_{j} ;
$$

then, summing over $j$ the expression

$$
\frac{1}{\theta}=\frac{\Pi_{j}}{\delta \epsilon_{j}}=\Theta \frac{\Pi_{j}}{\Theta} \frac{1}{\delta \epsilon_{j}},
$$

one finds

$$
\frac{N}{\theta}=\Theta\left\langle\delta \epsilon_{j}^{-1}\right\rangle
$$

As it is immediate to verify that $\left\langle\delta \epsilon_{g}^{-1}\right\rangle \geq\left\langle\delta \epsilon_{g}\right\rangle^{-1}$, where the equality sign holds only for $\delta \epsilon_{g j}$ all equal, write

$$
\left\langle\delta \epsilon_{g}^{-1}\right\rangle=\zeta\left\langle\delta \epsilon_{g}\right\rangle^{-1}, \quad \zeta \geq 1
$$

so

$$
\frac{N}{\theta}=\zeta \frac{\Theta}{\left\langle\delta \epsilon_{g}\right\rangle}
$$

reads

$$
N \frac{\left\langle\delta \epsilon_{g}\right\rangle}{\theta} \geq \Theta \text { i.e. } \frac{\delta S}{\Theta} \geq \frac{1}{N}, \quad \delta S=\frac{\left\langle\delta \epsilon_{g}\right\rangle}{\theta} .
$$

At the equilibrium, when all $\Pi_{j}$ are all equal to a unique $\Pi$ so that $\Theta_{e q}=N \Pi$ by definition, one finds once more

$$
\delta S \geq \Pi \text {. }
$$

Note that all $\theta \Pi_{0 j}-\varepsilon_{g j} \neq 0$ does not exclude $\left\langle\theta \Pi_{0 j}-\varepsilon_{g j}\right\rangle=0$; if so, according to (5.16) and (5.11) holds the equality sign that implies $\langle H-U\rangle=\langle L\rangle=0$ resulting from the possible chance $\left\langle\delta \epsilon_{g}\right\rangle=0$.

Here appears again, but now explicitly, that $\delta S \geq 0$ implies no work done on or performed by the system, which is one condition for an isolated system.

2) An interesting way to regard further (5.4) implements the following position

$$
\Theta \frac{\left\langle v_{g}^{2}\right\rangle}{c^{2}}=\sum_{j} \frac{\theta \delta S_{j}}{-\theta \log \Pi_{j} / \Pi_{0}}, \quad \delta \Pi_{j}=\Pi_{j}-\Pi_{0}
$$

and considers $\delta S_{j}>0$, so that by definition $\Pi_{j}<\Pi_{0}$. Unlike before in (5.6), 
now all $\Pi_{j}$ of $\delta \Pi_{j}$ are regarded with respect to a unique constant $\Pi_{0}$ that fulfills by definition the given inequality. Next introduce two arbitrary masses $m_{j}>m_{0}$ such that $m_{j} \Pi_{j}=m_{0} \Pi_{0}$, whence $\log \left(\Pi_{j} / \Pi_{0}\right)=\log \left(m_{0} / m_{j}\right)$. Then

$$
\log \frac{\Pi_{j}}{\Pi_{0}}=\log \frac{m_{0}}{m_{j}}=-\log \frac{m_{j} / V}{m_{0} / V}=-\log \frac{C_{j}}{C_{0}} ;
$$

this position introduces an arbitrary volume $V$ defining two densities $C_{0}$ and $C_{j}$ of the respective masses. So, owing to (3.1) it is possible to write (5.17) as

$$
\Theta \frac{\left\langle v_{g}^{2}\right\rangle}{c^{2}}=\sum_{j} \frac{\theta \delta S_{j}}{\mu_{j}}, \quad \mu_{j}=\theta \log \frac{C_{j}}{C_{0}}
$$

Consider now this result at the equilibrium with all $\Pi_{j} \equiv \Pi_{e q}$ and thus $C_{j} \equiv C$, while being also $\Theta_{e q}=N \Pi_{e q}$ by definition; in this particular but important case one finds owing to (5.18)

$$
N \Pi_{e q} \frac{\left\langle v_{g}^{2}\right\rangle_{e q}}{c^{2}}=\frac{\theta \delta S}{\mu}, \quad \mu=\theta \log C_{e q}+\text { const }, \quad \text { const }=-\theta \log C_{0}
$$

For example $C$ can be related to the number of $m o l$ in an ideal gas with $C=m o l / V=P / R T$, in which case $\mu$ takes the familiar form $\mu=\theta \log P$; of course $C_{0}$ is automatically to be regarded with reference to its coherent energy constant, which indeed is the so called "reference state".

So, now one finds the chemical potential $\mu$ as statistical average of all quantum microstates of the system. Also in this case is explicitly apparent in this result the implication

$$
\delta S \geq 0,
$$

where the equality sign holds for $C=C_{e q}=C_{0}$ : indeed in (5.17) is possible $\Pi_{j}=\Pi_{0}$ provided that $\delta S_{j}=0$ itself. As expected, this formulation of the second law describes explicitly the tendency of any system to flatten the local concentration/composition differences to reach the equilibrium state. In effect the different density distributions in a crystal lattice, due for example to local composition gradients, are intuitively a form of disorder with respect to the perfect order represented by its uniform composition. Hence $\delta S=S_{e q}-S_{i n}$ means that the equilibrium state is attained via an entropy increase with respect to an arbitrary initial disordered state.

\section{Statistical Distributions}

Let us check now the validity of (5.18) starting from the statistical Equation (1.6) regarded as a collective property of the system of particles. Since the second (1.3) has macroscopic statistical meaning, the inequality $S \leq \Theta$ written as

$$
\sum_{j} \Pi_{j}\left(1+\log \Pi_{j}\right) \geq 0
$$

must be fulfilled by the sum, not necessarily by all single terms $\Pi_{j}\left(1+\log \Pi_{j}\right)$ separately. In fact every $j$-th term alone fulfills 


$$
\Pi_{j}\left(1+\log \Pi_{j}\right) \geq 0
$$

for $\Pi_{j}>e^{-1}$ but not for $\Pi_{j}<e^{-1}$. On the one hand the chance that $\Theta>1$, in principle possible for $\Theta \neq 1$, does not prevent assuming all $\Pi_{j}>e^{-1}$; on the other hand, however, the physical validity of (6.1) is easily shown even regardless of whether actually some addends contribute to the sum with $\Pi_{j}<e^{-1}$. In other words it is possible to show that (6.2) is enough but not necessary to fulfill (6.1). Let indeed $1+\log \Pi_{j} \lessgtr 0$ be introduced now and expressed by

$$
1+\log \Pi_{j}=g_{j}, \quad g_{j} \lessgtr 0,
$$

with both signs allowed for the arbitrary function $g_{j}$ without violating (6.1) so that $\Pi_{j}=\exp \left(g_{j}-1\right)$. Next calculate $\delta \Pi_{j}=\exp \left(g_{j}-1\right) \delta g_{j}$, which reads

$$
\Pi_{j}=\exp \left(g_{j}-1\right) \delta g_{j} \mp \Pi_{0}
$$

once having written explicitly $\delta \Pi_{j}=\Pi_{j} \pm \Pi_{0}$ to which corresponds in effect an appropriate sign of $\delta g_{j}$. The double sign is justified by the chance of modifying $\delta \Pi_{j}$ increasing or decreasing its initial value $\Pi_{j}$. With the positions involving the number $N_{j}$, in agreement with the previous reasoning to infer (5.18),

$$
\frac{\Pi_{j}}{\Pi_{0}}=\frac{m_{0}}{m_{j}}=\frac{N_{0} m_{x}}{N_{j} m_{x}}=\frac{N_{0}}{N_{j}} ;
$$

i.e. via the total number $N_{0}$ of particles of the system and the number $N_{j}$ of particles in the $j$-th state, the Equation (6.4) yields

$$
\frac{\Pi_{j}}{\Pi_{0}}=\frac{N_{0}}{N_{j}}=\frac{\delta g_{j}}{\Pi_{0}} \exp \left(g_{j}-1\right) \mp 1 .
$$

Hence rewriting

$$
\frac{\delta g_{j}}{\Pi_{0}}=\exp \left(s_{j}-s_{0}+1\right),
$$

where $s_{j}$ and its corresponding $s_{0}$ are related to the arbitrary $\delta g_{j}$, the last expression turns with trivial manipulations into

$$
N_{j}=\frac{N_{0}}{\exp \left(r_{j}-s_{0}\right) \mp 1}, \quad r_{j}=g_{j}+s_{j}>0
$$

whatever the sign of $g_{j}$ might be thanks to the additional term $s_{j}$ coming from $\delta g_{j}$. Is evident the physical meaning of this result regarding the dimensionless $r_{j}-s_{0}$ as $\left(\varepsilon_{j}-\varepsilon_{0}\right) / \theta$, as done in (5.5) and (5.7). This well known equation was obtained in [1] following a different reasoning, through which the link between spin and statistical distribution has been also concerned. Here further considerations are superfluous; is however essential the fact of having found the quantum statistical distributions regardless of the single values of $\Pi_{j}$ that determine the signs of the respective terms of (6.1) consistent with (6.3). These signs do not prevent the physical meaning of the whole sum, thus confirming that actually (1.6) holds considering the sum of all states. From a physical point of view the validity of (6.5) means that in a system of particles are 
allowed states of local order and disorder without contradicting (1.3) of the whole system.

\section{Quantum Correspondences: Bell Inequality}

After having introduced a self consistent landscape of relativistic properties of thermodynamic functions linking macro and quantum micro states of physical systems, is justified the further extension of these results to better emphasize the quantum basis unifying all concepts of the present theoretical model.

Consider (1.4) recalling that all terms have probabilistic meaning and range as in (1.3); write then (1.4) as

$$
S+S^{o}=\Theta=-\sum_{j=1}^{N} \Pi_{j} \log \Pi_{j}-\sum_{j=1}^{N} \Pi_{j} \frac{\delta S_{j}}{\delta \Pi_{j}} .
$$

This equation can be identically rewritten as

$$
S+S^{o}=\Theta=-\sum_{j=1}^{j_{d}} \Pi_{j} \log \Pi_{j}-\sum_{j=j_{d}+1}^{N} \Pi_{j} \log \Pi_{j}-\sum_{j=1}^{j_{o}} \Pi_{j} \frac{\delta S_{j}}{\delta \Pi_{j}}-\sum_{j=j_{o}+1}^{N} \Pi_{j} \frac{\delta S_{j}}{\delta \Pi_{j}},
$$

where the summations over all allowed states have been simply split into partial sums defined by the arbitrary limits $j_{d}$ and $j_{o}$. Next, it is convenient to rewrite again this equation as

$$
S+S^{o}=\Theta=S_{d}+\delta \Theta^{*}+S_{o},
$$

where

$$
\begin{aligned}
& S_{d}=-\sum_{j=1}^{j_{d}} \Pi_{j} \log \Pi_{j}, \quad S_{o}=-\sum_{j=1}^{j_{o}} \Pi_{j} \frac{\delta S_{j}}{\delta \Pi_{j}}, \\
& \delta \Theta^{*}=-\sum_{j=j_{d}+1}^{N} \Pi_{j} \log \Pi_{j}-\sum_{j=j_{o}+1}^{N} \Pi_{j} \frac{\delta S_{j}}{\delta \Pi_{j}} .
\end{aligned}
$$

The notation is justified because $\delta \Theta^{*}$ reads

$$
\delta \Theta^{*}=-\sum_{j=j_{d}+1}^{N}\left(\Pi_{j} \log \Pi_{j}+\Pi_{j} \frac{\delta S_{j}}{\delta \Pi_{j}}\right)-\sum_{j=j_{o}+1}^{j_{d}} \Pi_{j} \frac{\delta S_{j}}{\delta \Pi_{j}}=\sum_{j=j_{d}+1}^{N} \Pi_{j}-\sum_{j_{o}+1}^{j_{d}} \Pi_{j} \frac{\delta S_{j}}{\delta \Pi_{j}}
$$

that in turn yields

$$
\begin{aligned}
\delta \Theta^{*} & =\sum_{j=j_{d}+1}^{N} \Pi_{j}-\sum_{j_{o}+1}^{j_{d}} \Pi_{j} \frac{\delta S_{j}}{\delta \Pi_{j}}=\sum_{j=1}^{N} \Pi_{j}-\sum_{j=1}^{j_{d}} \Pi_{j}-\sum_{j_{o}+1}^{j_{d}} \Pi_{j} \frac{\delta S_{j}}{\delta \Pi_{j}} \\
& =\Theta-\sum_{j=1}^{j_{d}} \Pi_{j}-\sum_{j_{o}+1}^{j_{d}} \Pi_{j} \frac{\delta S_{j}}{\delta \Pi_{j}} ;
\end{aligned}
$$

i.e. $\delta \Theta^{*}=\Theta-\Theta^{*}$, where $\Theta^{*}$ summarizes both addends in the last equality (7.4). Therefore subtracting $S_{d}+S_{o}+\delta \Theta^{*}-\Theta=0$ from $S+S^{o}-\Theta=0$ according to (7.2), one finds

$$
\Delta S+\Delta S^{o}-\delta \Theta^{*} \geq 0, \quad \Delta S=S-S_{d}, \quad \Delta S^{o}=S^{o}-S_{o} .
$$

With these premises, it this possible to highlight more Expressively (7.5) introducing Bell's language: the aim is to show without "ad hoc" hypotheses that 
the second law $\delta S \geq 0$, previously obtained in (3.15) and (5.19) via thermodynamic considerations, is actually a Bell inequality. Are available here three independent and arbitrary parameters: $j_{d}$ and $j_{o}$, plus $N$ that will be in fact implemented as a third independent parameter $N-j_{d}$.

1) Write first

$$
\Delta S=S-S_{d}=\Pi(X, N)-\Pi\left(X, j_{d}\right):
$$

the notation emphasizes that $S$ depends on an appropriate parameter $X$, which reasonably is a representative feature of the specific system; e.g. $X$ depends on whether the system is crystal lattice or gas or liquid or amorphous material. The notation $\Pi\left(X, j_{d}\right)$ for $S_{d}$ is quite obvious. Take $N$ as a fixed parameter, which for simplicity of notation will be thus omitted in the following, whereas instead $j_{d}$ and $j_{o}$ affect of course the resulting $\Delta S$ concerned here. Note that

$$
\Pi(X, n o t Z)=\Pi(X, n o t Z)+\Pi(X, Z)-\Pi(X, Z)=\Pi(X)-\Pi(X, Z):
$$

in general, indeed, it is intuitive that the sum of probabilities $\Pi(X, n o t Z)$ plus $\Pi(X, Z)$ reduces to $\Pi(X)$ merging both possible chances not $Z$ and $Z$. So, summing and subtracting $\Pi(X, Z)$, the difference of probabilities at the right hand side is summarized by the unique not term at the left hand side. Thus, whatever $X$ might be,

$$
\Delta S=S-S_{o}=\Pi(X, n o t Z), \quad Z \rightarrow j_{d}, \quad n o t Z \rightarrow N-j_{d} .
$$

2) An analogous reasoning holds to handle the term

$$
\Delta S^{o}=S^{o}-S_{o}=\Pi(Y, X)-\Pi\left(Y, j_{o}\right) ;
$$

in effect one finds now

$$
\Pi(Y, \operatorname{not} X)=\Pi(Y, \operatorname{not} X)+\Pi(Y, X)-\Pi(Y, X)=\Pi(Y)-\Pi(Y, X) .
$$

Hence, owing to the previous considerations,

$$
\Delta S^{o}=\Pi(Y, n o t X), \quad X \rightarrow j_{o}, \quad n o t X=N-j_{o} .
$$

3) As concerns $\delta \Theta^{*}$, it is positive owing to (7.3) and (5.1). Considering that $\Theta^{*}$ consists of terms from $j=j_{d}$ to $j=N$, regard then $\delta \Theta^{*}=\delta \Theta^{*}\left(N-j_{d}\right)$ and thus

$$
\Pi(Y, n o t Z)=\Pi(Y, n o t Z)+\Pi(Y, Z)-\Pi(Y, Z)=\Pi(Y)-\Pi(Y, Z) ;
$$

hence

$$
\delta \Theta^{*}=\Theta-\Theta^{*}=\Pi(Y)-\Pi(Y, Z)=\Pi(Y, \operatorname{not} Z), \quad Y=N .
$$

In summary, Collecting (7.6), (7.7) and (7.8), by comparison with (7.5) it is possible to write three possible correspondences linking the three ranges (7.5) to the respective not. So from

$$
\begin{aligned}
& \Delta S \quad+\Delta S^{o} \quad-\quad \delta \Theta^{*} \geq 0 \\
& \uparrow \\
& \Pi(X, \operatorname{not} Z)+\Pi(Y, \operatorname{not} X)-\Pi(Y, \operatorname{not} Z) \geq 0
\end{aligned}
$$


follows therefore the correlation

$$
\Pi(X, \operatorname{not} Z)+\Pi(Y, n \operatorname{not} X)-\Pi(Y, \operatorname{not} Z) \geq 0 \leftrightarrow \delta S \geq 0 .
$$

The former is in fact the Bell inequality, usually written

$$
\text { Number }_{1}(X, \text { notZ })+\text { Number }_{2}(Y, \text { not } X)-\text { Number }_{3}(Y, \text { notZ }) \geq 0
$$

here normalized dividing both sides by Number $_{1}+$ Number $_{2}+$ Number $_{3}$; in this way the three Number turn the addends into the respective probabilities. The correspondence (7.10) with the second law is clear: (7.9) comes from (7.2) that simply rewrites (7.1); in turn this latter is straightforward consequence of (1.3) from which has been obtained $\delta S \geq 0$, see eqs (3.15) and (5.19).

In other words, the Equation (1.3) imply the second law both in its usual entropic form when handled as shown in section 5 and equivalently in the Bell form when handled as just shown here. Therefore (3.15) and (5.19) already assessed via reliable thermodynamic considerations are confirmed through a sound quantum reason, the Bell inequality.

A crucial aspect of this result is that $\delta S \geq 0$ is calculated as difference between $S$ of a system with $N$ states and another $S_{d}$ of a system with $j_{d} \leq N$ allowed states; this shows that in fact the entropy tends to increase along with the number of states, in turn corresponding to an increased total disorder of the whole system. Obviously the positions (7.2) concern an isolated system, because they do not involve explicitly any external energy that could modify them: in effect according to (7.5) all addends are self-defined uniquely through the allowed states of the system, regardless of any possible external energy, $\mathcal{L}$ or $\mathcal{Q}$, that in principle can modify the spontaneous evolution of the system explicitly inherent the right hand side of (5.8) and (5.9).

It is known that the Bell inequality is the key to prove the non locality and reality of the quantum world; hence, recalling also the previous results, the correlations (1.5) are to be considered at this point definitively proven.

The correlations (TP0) deserve a further comment.

Encouraged by this result let us check the chance of linking to the Bell formalism also the Pauli principle that in effect reads $N_{p}=1$ or $N_{p} \geq 1$, being $N_{p}$ the number of particles allowed per quantum state; here we have omitted $N_{p}=0$, that trivially means empty quantum state. It is known that the equality sign only holds for fermions, whereas the inequality holds for bosons. The following simple considerations show also this result.

Add and subtract $\Pi(X, Z)$ at left hand side of the first (7.10). So

$$
\Pi(Y, \operatorname{not} X)+\Pi(X, \operatorname{not} Z)-\Pi(Y, \operatorname{not} Z)-\Pi(Y, Z)+\Pi(Y, Z) \geq 0
$$

i.e.

$$
\Pi(Y, \operatorname{not} X)+\Pi(X, \operatorname{not} Z)-\Pi(Y)+\Pi(Y, Z) \geq 0,
$$

which reads

$$
\Pi(Y, n o t X)+\Pi(X, n o t Z)+\Pi(Y, Z) \geq 0+\Pi(Y), \quad \Pi(Y)=\Pi(Y, n o t Z)+\Pi(Y, Z) .
$$


Now introduce the new condition that two chances only are allowed for $Z$, i.e. $Z=Z_{e}$ or $Z=Z_{o}$; so not $Z$ turns these $Z$-s respectively into not $Z=Z_{o}$ and $n o t Z=Z_{e}$ where the subscripts stand for "even" and "odd". In other words not exchanges the states labeled with subscripts $e$ and $o$. Consider the case where the system is formed by particles of one kind only fulfilling either chance; then one expects two corresponding inequalities

$$
\begin{aligned}
& \Pi(Y, \operatorname{not} X)+\Pi\left(X, Z_{e}\right)+\Pi\left(Y, Z_{o}\right) \geq 0+\Pi(Y), \\
& \Pi(Y, \operatorname{not} X)+\Pi\left(X, Z_{o}\right)+\Pi\left(Y, Z_{e}\right) \geq 0+\Pi(Y) .
\end{aligned}
$$

Summing these inequalities one finds

$$
2 \Pi(Y, n o t X)+\Pi\left(X, Z_{e}\right)+\Pi\left(Y, Z_{o}\right)+\Pi\left(X, Z_{o}\right)+\Pi\left(Y, Z_{e}\right) \geq 0+2 \Pi(Y) ;
$$

thus, being

$$
\begin{aligned}
& \Pi\left(X, Z_{e}\right)+\Pi\left(X, Z_{o}\right)=\Pi(X), \\
& \Pi\left(Y, Z_{o}\right)+\Pi\left(Y, Z_{e}\right)=\Pi(Y)
\end{aligned}
$$

for the aforesaid reasons, (7.13) reads

$$
2 \Pi(Y, n o t X)+\Pi(X)+\Pi(Y) \geq 0+2 \Pi(Y)
$$

i.e.

$$
\Pi(Y, \operatorname{not} X) \geq 0+\frac{\Pi(Y)-\Pi(X)}{2} .
$$

Then

$$
\Pi(Y, \operatorname{not} X)=\Pi(Y)-\Pi(Y, X) \geq \frac{\Pi(Y)-\Pi(Y, X)}{2} .
$$

Considering the inequality symbol, the validity of this result is self evident whatever $\Pi(Y)$ and $\Pi(Y, X)$ might be; in this case no further condition is required. To fulfill the equality, instead, a glance to (7.15) reveals that (7.15) can be fulfilled if both sides are anyway equal to zero only. This is possible either if $\Pi(Y)=\Pi(Y, X)=0$ that in turn requires $X=Y=0$, or if a couple $X^{*} \neq 0$ and $Y^{*} \neq 0$ exists such $\Pi\left(Y^{*}\right)=\Pi\left(Y^{*}, X^{*}\right)$. Thus are possible two chances only: the former trivially means that the probability of occupancy of a given quantum state is identically null, empty state, the latter that admits the occupancy of a particle whose $X$ and $Y$ are uniquely selected, $X=X^{*}$ and $Y=Y^{*}$, compatible with probabilities $\Pi\left(Y^{*}\right)$ and $\Pi\left(Y^{*}, X^{*}\right)$ non identically null but equal. In fact is in principle admissible one well specified couple of values $X^{*}$ and $Y^{*}$ : according to (7.7) and (7.8), indeed, $j_{o}=1,2,3, \cdots, N$ and thus $N-j_{o}=N, N-1, N-2, N-3, \cdots, 2,1,0$, so that the first and unique occupancy allowed beside the empty state corresponds to nothing else but $N-j_{o}^{*}=X^{*}$ to which is related just $X^{*}$ for a given $Y^{*}=N$; moreover $X^{*}$ is unique because but next, since the value of $X=N-j_{o}$ are all different. So one must conclude that the occupancy numbers allowed by the equality are 0,1 only already introduced, whatever other possible $Y$ and $X$ might be.

This is clearly the Pauli principle: although the Bell inequality does not 
concern explicitly the existence of a property of particles we call "spin", nevertheless it states the principle according which in Nature must exist two ways to fill the allowed states of a physical system. Regarding $Z$ as a two valued property compliant with (7.12) would seem seemingly a weird "ad hoc" hypothesis, but knowing the existence of the spin this position becomes a fundamental statement to acknowledge the two different ways to fill the allowed states of physical systems in agreement with (6.5). Also this point has been better explained in [1].

\section{Further Implications of (1.5)}

If it is true that the entropy is the "arrow of time", then once more (1.5) should be adequate to infer an interesting implication: to describe the evolution of the Universe. This is the purpose of this last section.

Rewrite the previous (3.7) as follows

$$
\delta \ell^{2}=c^{2} \delta t^{2}-\delta s_{g}^{2}=c^{2} \delta t^{\prime 2}-\delta s_{g}^{\prime 2}=\frac{c^{2} \delta t^{2}}{\gamma^{2}}-\gamma^{2} \delta s_{g}^{2}:
$$

the first equality reminds the starting point (3.7) in $R$, the second equality is (3.7) with time and space ranges $\delta t^{\prime}$ and $\delta s_{g}^{\prime}$ defined in another inertial reference system $R^{\prime}$, the third equality rewrites the second one emphasizing explicitly time dilation and space contraction of the initial respective ranges in $R$ with respect to that in $R^{\prime}$. Implement now the new position

$$
\chi^{2}=1-\frac{v_{m}^{2}}{c^{2}}=\frac{q_{0}}{\gamma^{2}}
$$

that introduces the function $\chi=\chi\left(v_{m}, q_{0} / \gamma^{2}\right)$ of $\gamma^{2}$ as shown via the factor $q_{0}$ and velocity $v_{m}$ to be defined. In fact (8.2) simply replaces $v_{g}$ of $\gamma^{2}$ in (3.6) with a new velocity $v_{m}^{2}$; its form has been guessed in order to write with the help of (1.5) and (1.3)

$$
\frac{q_{0}}{\gamma^{2}}+\frac{v_{m}^{2}}{c^{2}}=\frac{S}{\Theta}+\frac{S^{o}}{\Theta}=\frac{m_{r}^{2}}{m^{2}}+\frac{v_{g}^{2}}{c^{2}}=1, \quad \frac{m_{r}^{2}}{m^{2}}=\gamma^{2}, \quad q_{0}<1 ;
$$

so, replacing in (8.1) $\gamma^{2}=q_{0} / \chi^{2}$ according to the last equality, $\delta \ell^{2}$ becomes

$$
\begin{aligned}
& \delta \ell^{\prime \prime 2}=\left(\chi^{2} / q_{0}\right) / c^{2} \delta t^{2}-\frac{\delta s_{g}^{2}}{\chi^{2} / q_{0}}=\left(1-v_{m}^{2} / c^{2}\right) c^{2} \delta t^{\prime \prime 2}-\frac{\delta s_{g}^{\prime \prime 2}}{1-v_{m}^{2} / c^{2}}, \\
& \delta s_{g}^{\prime \prime 2}=q_{0} \delta s_{g}^{2}, \quad \delta t^{\prime \prime 2}=\frac{\delta t^{2}}{q_{0}} .
\end{aligned}
$$

To understand the last equality (8.2) that defines $v_{m}$ in the particular case of an attractive gravity field, remember (4.6) and multiply both sides by an arbitrary mass $m^{\prime}$ so that

$$
\frac{1}{2} m^{\prime} v_{m}^{2} \approx \frac{m^{\prime} m G}{\delta r} \text { i.e. } v_{m}^{2}=\frac{2 m G}{\delta r}:
$$

the left hand side shows the kinetic energy of the mass $m^{\prime}$ in the gravitational 
field of $m$. In effect $v_{m}=\sqrt{2 m G / \delta r}$ is the classical escape velocity of $m^{\prime}$ at distance $\delta r$ from the gravity center of $m$; it holds for any $\delta r \geq r_{0}$, being $r_{0}$ the radius of $m$. Hence (8.4) takes the more familiar form

$$
\delta \ell^{\prime \prime 2}=\left(1-2 m G / c^{2} \delta r\right) c^{2} \delta t^{\prime 2}-\frac{\delta s_{g}^{\prime \prime 2}}{1-2 m G / c^{2} \delta r}
$$

i.e., taking the average of both sides and recalling (CC1),

$$
\left\langle\delta \ell^{\prime \prime 2}\right\rangle=\left\langle\ell^{\prime \prime 2}\right\rangle-\left\langle\ell^{\prime \prime}\right\rangle^{2}=\left\langle\left(1-2 m G / c^{2} \delta r\right) c^{2} \delta t^{\prime \prime 2}\right\rangle-\left\langle\frac{\delta s_{g}^{\prime \prime 2}}{1-2 m G / c^{2} \delta r}\right\rangle .
$$

In turn this result reads

$$
\left\langle\ell^{\prime \prime 2}\right\rangle=\left\langle\ell^{\prime \prime}\right\rangle^{2}+\left\langle\left(1-2 m G / c^{2} \delta r\right) c^{2} \delta t^{\prime \prime 2}\right\rangle-\left\langle\frac{\delta s_{g}^{\prime \prime 2}}{1-2 m G / c^{2} \delta r}\right\rangle,
$$

being

$$
\left\langle\ell^{\prime \prime}\right\rangle^{2}=\left\langle\ell^{\prime \prime}\right\rangle^{2}\left(\vartheta, \phi, \delta s_{g}^{\prime \prime 2}, \delta r^{2}, \delta t^{2}\right), \quad\left\langle\ell^{\prime \prime}\right\rangle^{2}=\left\langle\ell^{\prime \prime}\right\rangle^{2}\left(\vartheta, \phi, \delta s_{g}^{\prime \prime 2}, \delta r^{2}, \delta t^{2}\right)
$$

average functions of the arguments; regarding $\left\langle\delta \ell^{\prime 2}\right\rangle$ according to the quantum uncertainty, i.e. as an uncertainty range, its upper and lower boundaries $\left\langle\ell^{\prime \prime 2}\right\rangle$ and $\left\langle\ell^{\prime \prime}\right\rangle^{2}$ are in fact arbitrary. One recognizes in (8.6) the metric of the general relativity, which however here has a probabilistic character, once acknowledging at the right hand side of (8.5) an appropriate rotational term $\mathcal{R}=-\delta r^{2}\left(\sin ^{2} \vartheta \delta \phi^{2}+\delta \vartheta^{2}\right)$ that describes through $\left\langle\ell^{\prime \prime}\right\rangle^{2}$ the uniform reciprocal rotation of non-inertial reference systems; this term can be inferred in an elementary way. On the one hand the last equality (8.1) is the key to extend the invariant interval of special relativity to the metrics of general relativity; in fact it replaces $\gamma^{2}$ with $q_{0} / \chi^{2}$ that introduces the presence of mass in (3.7). On the other hand however $\left\langle\ell^{\prime \prime}\right\rangle^{2}$ is positive by definition, so it cannot account for the negative sign of $\mathcal{R}$ in (8.6); then some additional positive contribution $\mathcal{X}$ is missing in Einstein's early metric of standard general relativity such that actually $\left\langle\ell^{\prime \prime}\right\rangle^{2}=\mathcal{X}+\mathcal{R}>0$. Work in progress and previous results [8] indicate that this required contribution $\mathcal{X}$ has to do with the consequent cosmological constant, which therefore appears as a necessary correction to the early formulation of the theory and not as "ad hoc" hypothesis.

Since according to (8.2) and first (1.5) $\chi^{2} / q_{0}=\gamma^{-2}=\Theta / S$, (8.4) reads

$$
\delta \ell^{\prime \prime 2}=(\Theta / S) c^{2} \delta t^{2}-\frac{\delta s_{g}^{2}}{\Theta / S}=\left(1-v_{m}^{2} / c^{2}\right) c^{2} \delta t^{\prime \prime 2}-\frac{\delta s_{g}^{\prime \prime 2}}{1-v_{m}^{2} / c^{2}} ;
$$

thus, since

$$
(\Theta / S)^{2} c^{2} \delta t^{2}-\delta s_{g}^{2} \geq 0
$$

it must be true that

$$
(\Theta / S) c \delta t-\delta s_{g}=K, \quad K \geq 0
$$

where $K$ is a positive quantity. So recalling that $S=\varepsilon / \theta$ and being $\theta=k T$, this result reads 


$$
T=\frac{\left(K+\delta s_{g}\right) \varepsilon}{k \Theta c} \frac{1}{\delta t}=\frac{K_{0}}{\delta t}:
$$

this expression yields $\theta$, i.e. the temperature, as a function of $\delta t$. As it seems appropriate to express explicitly this result as a function of $S$ write identically $S / \Theta=\varepsilon / \theta \Theta$ so that $1 / \Theta=\varepsilon / S \theta \Theta$; replacing in the last equation one finds

$$
T^{2}=\frac{\left(K+\delta s_{g}\right) \varepsilon^{2}}{c k^{2} S \Theta} \frac{1}{\delta t}=\frac{K_{S}^{2}}{\delta t}, \quad K_{S}^{2}=\frac{\left(K+\delta s_{g}\right) \varepsilon^{2}}{c k^{2} S \Theta} .
$$

Substantially Equations (8.9) and (8.10) are conceptually identical; (8.10) differs from (8.9) only for having explicitly expressed $\theta$ as a function of $S$. These relationships are easily verifiable taking tentatively, for a first preliminary assessment, $K_{0}=$ const or $K_{S}=$ const ; either of them should allow plotting $\theta=k T$ vs $\delta t$ to check the respective chance. To this purpose is useful a diagram of $T$ vs time elaborated by the Fermilab [6] [7]: if these considerations are correct, then this plot should fit reasonable the temperatures of expanding Universe at various milestones after the Big Bang. More specifically is useful a $\log \log$ plot of $\theta$ vs $\delta t$ : one should obtain linear plots $\log \theta=-\log \delta t+\log K_{0}$ or $\log \theta=-1 / 2 \log \delta t+\log K_{S}$ and verify whether in fact both formulas are correct or wrong. The Figure 1, formerly obtained in [9] via different reasoning based on an operative definition of space time, shows that in effect the entropy is

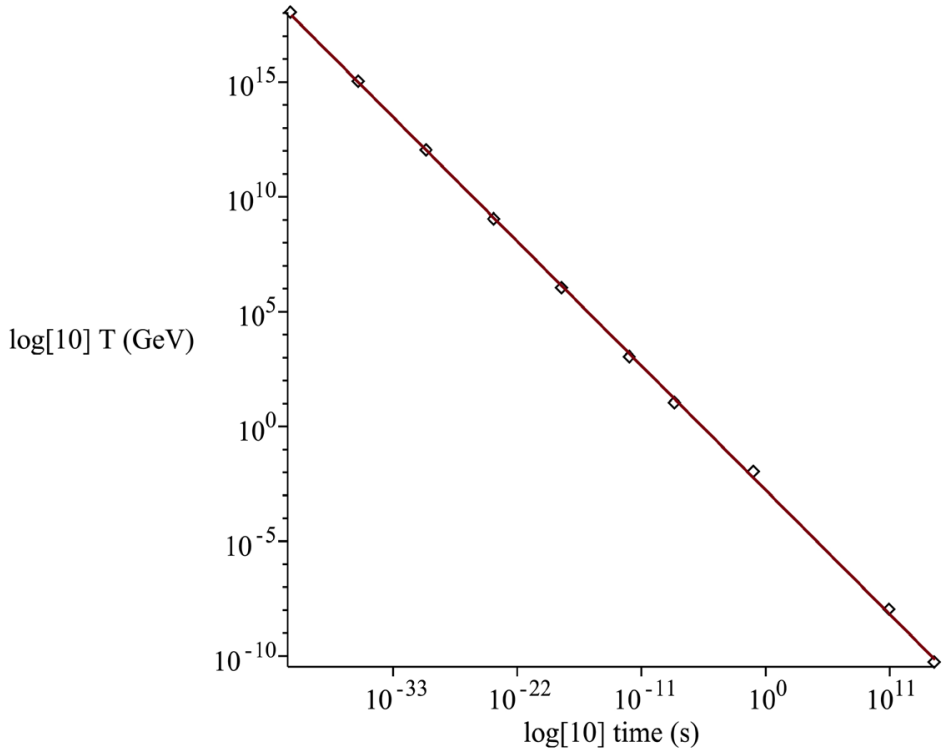

Figure 1. Timeline of the Universe comparing the plot calculated with (8.10) and the data of time and temperature shown in the plot authored by [6] and also reported in [7] since the Big Bang to today; $K_{s}$ has been preliminarily taken as a constant. This guess is sensible: the line represents the best fit regression $\log T=-0.492 \log \delta t-2.769$ of the data $(t, T)$ marked in the figure [6] by little arrows evidencing relevant events in the history of the Universe. The law revealed by this analysis, $T \propto \delta t^{-1 / 2}$, agrees with that found in [9] via different arguments not involving explicitly the entropy. 
the expected property that marks the evolution timeline of the Universe since its beginning to today. The best fit regression also shows that in fact (8.10) defines correctly the condition $K_{S}=$ const .

\section{Discussion}

The present paper must be regarded as an extension of the previous [1]. Most of the considerations hitherto carried out show that (1.4), formally legitimate, has actual physical worth and that its splitting into separate correlations (1.5) implies sensible outcomes. The probabilistic meaning of both (1.3) explains why (1.4) is more than mere algebraic unification of two equations seemingly different and highlights the correlations (1.5). Although (1.1) have been contextually inferred in the quoted paper through different arguments, it has been remarked for shortness in section 1 that the first (1.3) is simply the widely accepted velocity dependency of mass even though its inherent physical meaning concerns the actual probability that one particle exhibit dual corpuscular or wave like properties. The question about what has to do the wave/corpuscle dual behavior of a single particle with the probabilistic concept of order/disorder of a system of particles, has adequate statistical answer suggested by their form of alternative probabilities concurring to the respective certainties: the chance of quantum energy fluctuation implies actually both of them, as it appears in (1.1) through $m>m_{r}$ and in (3.11). The position (2.5) has made acceptable the idea of linking the average properties of a whole system to that of its $j$-th microstates, being these states representative of the macroscopic behavior even concerning one particle only as a limit case. In effect (1.4) regards dual behavior and entropy increase of matter as two different aspects of a unique probabilistic cause: the evolution of an undefined state merging both probabilities (1.3) brings to a new state revealed by and accessible to the experiment. So one particle appears as corpuscle or wave through its $m_{r} / m$ or $\left\langle v_{g}\right\rangle / c$ depending on what the experiment is aimed to measure; similarly where matter appears ordered or disordered with probabilities $S / \Theta$ or $S^{\circ} / \Theta$ via a microstructural observation. In fact, if the quantum world is non-local and non-real until an experiment is carried out, there is no reason to reject the idea that even order and disorder are undefined states that take physical meaning under the experiment. This consideration reminds for example Schrödinger's cat, with the dead cat described by $v \equiv\left\langle v_{g}\right\rangle \equiv 0$ to which corresponds dynamical mass $m$ equal to its rest mass $m_{r}$. This delicate conceptual point deserves special attention and explains the strategy followed in the previous and present paper: to reproduce well known results or infer crucial implications confirming the validity of (1.5) and related equations. Although several results have been already inferred in the quoted paper, it appeared appropriate to propose here further results to obtain more significant confirms.

The novelty of this study is the way to identify the common root underlying quantum physics, thermodynamics and relativity implementing the premises introduced in section 1; formulas and concepts are in turn part of a broader 
panorama of outcomes inferred in the holistic model [1] of evolving Universe. It is not surprising, therefore, the plot of Figure 1 involving directly the entropy.

Just in this respect the present model appears significant: a subtle connection is evidenced between quantum interpretation of De Broglie waves, statistical meaning of entropy and concept of invariance of the special relativity, which in turn implies Lorentz transformations, velocity dependence of mass and definition of thermodynamic functions. Also two results of general relativity, for brevity the Hawking entropy and the red shift only, have been included in section 4 as straightforward and natural corollaries of these premises. This connection compels accepting even the statistical meaning of invariant interval and Lorentz transformations evident since the early Equations (2.8) and (2.9). Consider in particular the entropy, which is known to be a fundamental principle of thermodynamics; it appears here closely related to other fundamental principles of Nature like the aforesaid dual behavior of matter and the Pauli principle via Bell's inequality. Thermodynamics was born as conceptual extrapolation of the technology of heat engines; with the contribution of the quantum theory, e.g. see Boltzmann's $H$ theorem and Maxwell equations, it became a fundamental Science itself. The present model introduces the basic principles of thermodynamics without implementing neither concepts of heat cycles nor intuitive assumptions like the energy conservation, in fact expressed through the first law. Actually it appears reductive to introduce fundamental concepts like $S$ and $\delta S \geq 0$ merely examining the Carnot cycle; as it is known that the Bell inequality is the key to prove the non locality and reality of the quantum world, (7.9) and (7.14) of the section 7 appear the most appropriate way to understand what really the entropy is and thus the correlations (1.5), which at this point are to be considered definitively proven.

\section{Conclusions}

The present model unifies within a common conceptual frame relativity, thermodynamics, quantum theory and, via the Bell inequality, dual wave/corpuscle behavior of matter and Pauli principle; electron diffraction, "Aufbau" of electrons in atoms and molecules and red shift appear rooted in a unique principle, the quantum uncertainty. Usually, the Bell inequality is synonym of EPR paradox; here however it appears as a law of thermodynamics.

In general, it is reductive and misleading to think quantum theory or relativity or thermodynamics as separate disciplines of physics; trying to merge all of them does not imply necessarily more difficult approach, but an in-depth investigation on their shared root. In effect, the laws of Nature reveal unexpected links and common implications that solve problems apparently different through simple arguments and, mostly important, elementary mathematical formalism.

\section{Conflicts of Interest}

The author declares no conflicts of interest regarding the publication of this 
paper.

\section{References}

[1] Tosto, S. (2018) Evolution, Quantization, Relativity: An Abinitio Model. Journal of Modern Physics, 9, Article ID: 89481.

[2] Landau, L. and Lifchitz, E. (1967) Physique Statistique. French, Ed., MIR Moscow.

[3] Planck, M. (1907) Zur Dynamik bewegter Systeme. Sitzungsberichte der Königlich-Preussischen Akademie der Wissenschaften, Berlin, Erster Halbband Vol. 29, 542-570.

[4] Hawking, S.W. (1974) Black Hole Explosions? Nature, 248, 30-31. https://doi.org/10.1038/248030a0

[5] Landau, L. and Lifchitz, E. (1966) Theorie du Champ. French, Ed. MIR Moscow.

[6] Rosenfeld, R. (2018) A Sketchy Introduction to Cosmology. School Proceedings, CYR, Vol. 4/2018, CERN-2018-007-SP. (CERN, Geneva)

[7] Kolb, E.W. and Turner, M.S. (1990) The Early Universe. Front. Phys., 69, 1.

[8] Tosto, S. (2016) Quantum and Relativistic Corollaries of an Operative Definition of Space Time. Journal of Advances in Physics, 11, 3408. https://doi.org/10.24297/jap.v11i5.362

[9] Tosto, S. (2013) Quantum Standpoint for a More Understandable Universe. American Journal of Space Science, 1, 22-32. https://doi.org/10.3844/ajssp.2013.22.32 